\title{
ENFERMEDAD DE ALZHEIMER Y DEGENERACIÓN MACULAR ASOCIADA A LA EDAD
}

\section{ALZHEIMER'S DISEASE AND AGE-RELATED MACULAR DEGENERATION}

\author{
ROCA-SANTIAGO HM ${ }^{1}$, LAGO-BOUZA JR ${ }^{2}$, MILLÁN-CALENTI JC ${ }^{2}$, \\ GÓMEZ-ULLA-IRAZAZÁBAL F²
}

\begin{abstract}
RESUMEN
Objetivos: Analizar diferentes factores de riesgo implicados en la aparición de la enfermedad de Alzheimer y de la degeneración macular asociada a la edad, tratando de establecer una relación de asociación entre ambas entidades.

Métodos: Sobre una muestra de 57 sujetos, de los que 33 presentan demencia (casos) y 24 no (controles) se realiza estudio analítico a fin de establecer el grado de asociación entre la enfermedad de Alzheimer (EA) y la degeneración macular asociada a la edad (DMAE). Para ello, tras realizar anamnesis a todos los sujetos, se estudia el fondo de ojo mediante cámara no midriática tipo Topcon TRV-50VT.

Resultados: De acuerdo a la hipótesis de trabajo planteada, aplicada la razón de productos cruzados o de disparidad (odds ratio) se obtuvo un resultado positivo que determina relación causa efecto, ya que el porcentaje de casos con DMAE $(42,4 \%)$ es superior al de controles con DMAE (25\%). Por otro lado mediante la Chi cuadrado de Pearson, aunque no se establecieron diferencias significativas, los datos obtenidos muestran protección en el grupo
\end{abstract}

\begin{abstract}
Objectives: We analysed different risk factors involved in the onset of both Alzheimer's disease (AD) and age-related macular degeneration (ARMD). The putative relation between both disorders was studied.

Methods: We studied 57 subjects to determine the correlation between AD and ARMD. Thirty-three of the subjects suffered dementia (cases), whereas 24 of them (controls) did not. Firstly, anamnesis was performed for all individuals. We then examined the macular area of the eye using a non-midriatic TRV-50VT fundus camera. Significant odds ratio $(\mathrm{OR})$ results were used as a statistical tool to survey the putative link between AD and ARMD.

Results: The OR test results showed that ARMD was associated with Alzheimer's disease. The occurrence of ARMD was significantly higher for cases $(42.4 \%)$ than for controls (25\%). On this basis, we inferred a cause-effect relation linking both variables. Our dataset suggested that the control group was more protected against ARMD than the case group, as revealed by Fisher's exact test $(\mathrm{P}=0.649)$.
\end{abstract}

Recibido: 8/6/05. Aceptado: 20/2/06.

Grupo de Investigación en Gerontología (GIG), Departamento de Medicina, Universidad de La Coruña, España.

Unidad de Retina y Diabetes Ocular, Facultad de Medicina, Universidad de Santiago de Compostela. España.

1 Licenciada en Medicina.

2 Doctor en Medicina.

Correspondencia:

F. Gómez-Ulla Irazazábal

Facultad de Medicina

C/. San Francisco

15782 Santiago de Compostela

España

E-mail: ciulla@usc.es 
control de acuerdo al estadístico exacto de Fisher $(\mathrm{p}=0,649)$.

Conclusiones: Existen evidencias suficientes para pensar que la EA y la DMAE podrían tener factores etiológicos y patogénicos comunes, y aunque en nuestro estudio no hemos podido establecer la relación significativa entre ambas patologías, posiblemente en base al tamaño de la muestra o a las diferencias de edades entre ambos grupos, creemos establecida la posible asociación como para plantear un futuro trabajo que pueda confirmar estos hallazgos.

Palabras clave: Enfermedad de Alzheimer, degeneración macular asociada a la edad, demencia, drusas.
However, such a difference between both groups was not strongly supported.

Conclusions: We suggest that AD and ARMD may have common factors concerning etiology and pathogenesis. Our dataset did not allow us to show a significant relation between both disorders, which is likely due to sample size and/or to age differences in the two studied groups. Even so, we feel that the possibility of such an association is justified, and future surveys to test this possibility are warranted. (Arch Soc Esp Oftalmol 2006; 81: 73-78).

Key words: Alzheimer's disease, age-related macular degeneration, neurodegeneration, retinal drusen.

\section{INTRODUCCIÓN}

En la actualidad las demencias representan la tercera causa de mortalidad, tras las enfermedades cardiovasculares y el cáncer, siendo la Enfermedad de Alzheimer (EA) la más frecuente de ellas. Las demencias constituyen uno de los problemas sociosanitarios más relevantes por su impacto social de irreversibilidad, el desconocimiento de sus factores de riesgo con un reducido margen de prevención y el rápido aumento de su incidencia de acuerdo al incremento de la esperanza de vida en las sociedades desarrolladas. Por otro lado, las demencias, generan una gran repercusión sobre el gasto generado, que va a crecer de forma exponencial a medida que la enfermedad progrese.

La degeneración macular asociada a la edad (DMAE), descrita por primera vez por Pagenstecher y Gente en 1876, suele iniciar sus manifestaciones por encima de la sexta década. A nivel del fondo de ojo, se observan depósitos blanco-amarillentos (drusas), que acaban alterando la interfaz coroides-retina afectando la irrigación de los fotorreceptores y, en muchos casos, dependiendo del tipo, confluencia, tamaño y localización de las drusas respecto a la fóvea, pueden degenerar o evolucionar hacia una neovascularización coroidea (DMAE neovascular) o placa de atrofia (DMAE atrófica). La DMAE es la principal causa de ceguera legal en los países desarrollados.

La mayoría de los estudios $(1,2)$ coinciden en que el riesgo de desarrollar DMAE aumenta con la edad, estimando la OMS (3) que la población de 60 o más años de edad se duplicará durante los próximos 20 años.

Algunos autores (4-6) han tratado de asociar la EA y la DMAE en base a sus características genéticas, ya que ambos procesos son considerados desórdenes neurodegenerativos crónicos con características comunes como pueden ser su relación con el envejecimiento, su etiología desconocida y más específicamente la presencia de placas seniles (formaciones extracelulares con un núcleo interno de fibras de péptido amiloide $A \beta$ ) tanto en la sustancia gris cerebral como en la mácula de la retina. Además, a nivel histológico en la EA van a aparecer ovillos neurofibrilares (agregados de la proteína TAU hiperfosforilada), junto con otras alteraciones menos específicas.

Aunque la etiología de ambas patologías no está claramente establecida, se sabe que su origen es multicausal, tanto en base a factores genéticos como adquiridos. Entre los factores genéticos (7-9) relacionados con la EA se encuentran la historia familiar, la mutación precoz del gen de la proteína precursora de amiloide (APP) o de la presenilina 1 (PS1) o presenilina 2 (PS2) o el genotipo ApoE (alelos $\mathrm{E}_{2}, \mathrm{E}_{3}, \mathrm{E}_{4}$ ), implicado en el transporte del colesterol, con acciones diferentes ya que mientras la forma alélica E4 incrementa el riesgo de padecer la EA, el alelo E2 parece proteger de la enfermedad $(8,9)$; siendo factores adquiridos, la edad, el sexo femenino, la dieta hipercalórica y rica en ácidos grasos saturados y la obesidad. Con respecto a la DMAE, son factores de riesgo relacionados con su 
aparición, la raza blanca, edad avanzada, sexo femenino, hábito tabáquico, hipercolesterolemia, la exposición solar, ojos claros, hipermetropía, cirugía previa de cataratas, además de los antecedentes familiares (tabla I).

Ambas patologías, EA y DMAE, van a coincidir en su repercusión sobre la calidad de vida del enfermo, aumentando el riesgo de caídas y de aislamiento social que en muchos casos generará un cuadro de depresión clínica establecida.

Como hipótesis de trabajo nos planteamos establecer la relación entre la EA y la DMAE en base a criterios neurodegenerativos; siendo objetivos a conseguir el establecer la posible asociación entre ambas patologías que nos permita elaborar criterios de diagnóstico precoz de EA a partir de las lesiones degenerativas del fondo de ojo (DMAE).

\section{SUJETOS, MATERIAL Y MÉTODO}

Se realiza estudio analítico, no experimental con sentido retrospectivo sobre una muestra no probabilística dividida en dos grupos (casos y controles), uno compuesto por 33 sujetos ambulatorios (casos) que acuden a un centro especializado en la atención a personas con dependencia, con una edad media de $79,58$ años (DT $\pm 8,05)$, de los que $20(60,6 \%)$ fueron mujeres y 13 hombres $(39,4 \%)$; y otro compuesto por 24 individuos (controles) no institucionalizados, sin diagnóstico conocido de patologías degenerativas, con una edad media de 71 años $(\mathrm{DT} \pm 5,78)$, de los que $16(66,7 \%)$ fueron mujeres $\mathrm{y}$ $8(33,3 \%)$ hombres.

Para el grupo de casos se utilizaron como criterios de inclusión; el ser mayores de 60 años, estar

Tabla I. Factores de riesgo implicados en cada una de las patologías estudiadas. EA y DMAE

\begin{tabular}{ll}
\hline \multicolumn{1}{c}{ EA } & \multicolumn{1}{c}{ Factores de riesgo } \\
& \\
\hline Antecedentes familiares & Antecedentes familiares \\
Edad & Edad \\
Sexo femenino & Sexo femenino \\
Raza blanca & Raza blanca \\
Enf. Cardiovasculares (HTA) & Enf. Cardiovasculares (HTA) \\
Hipercolesterolemia & Hipercolesterolemia \\
Tabaco & Tabaco \\
Depresión & Exposición solar \\
Proteína precursora de amiloide & Ojos claros \\
Genotipo Apo E & Cirugía previa de cataratas \\
\hline \hline
\end{tabular}

diagnosticados de EA según los criterios NINCDSADRDA (10) y del DSM-IVR (11) o de DMAE de Bird y Wisconsin (12), el haber dado el consentimiento informado el paciente, o bien en caso de incapacitación el responsable y el de querer participar voluntariamente en el estudio. Mientras que fueron criterios de exclusión el padecer EA moderada-grave que impidiera la realización de la exploración o miosis bilateral (pupila con un diámetro menor de $2 \mathrm{~mm}$ sin reflejo fotomotor que lo justifique), de acuerdo al propio envejecimiento o al consumo de fármacos.

Se realiza historia clínica completa, que en el grupo casos se cumplimenta con la ayuda del familiar, allegado o cuidador principal, en la que se recogen datos referidos a la exposición a los factores de riesgo descritos para ambas patologías, así como antecedentes familiares y antecedentes clínico-patológicos, entre otros. A continuación, se realiza estudio del fondo de ojo tomando una retinografía en $50^{\circ}$ centrada en la mácula de cada ojo con cámara no midriática Topcon TRV-50VT.

Con respecto al grupo control, fueron criterios de inclusión el ser mayores de 60 años y no haber sido diagnosticados previamente de EA o DMAE.

La información ha sido sistematizada y analizada en el programa estadístico SPSS versión 11.5 para Windows, utilizándose, a fin de establecer la asociación entre la EA y la DMAE, el estadístico chicuadrado de Pearson (Odds ratio).

\section{RESULTADOS}

De los 33 sujetos adscritos al grupo de casos, 28 $(84,8 \%)$ estaban diagnosticado de demencia tipo Alzheimer y $5(15,2 \%)$ de otras demencias, presentando DMAE 14 (42,4\%), de los cuales $12(36,6 \%)$ tienen además EA. Del grupo control, únicamente 6 sujetos presentaron DMAE (24\%).

Atendiendo a los factores de riesgo implicados en cada una de las patologías del grupo casos, un $60,71 \%$ de los sujetos diagnosticados de demencia presentaban hipertensión arterial y un 64,28\% presentaban valores de colesterol en sangre elevados. De los sujetos con DMAE, un 60\% refieren exposición solar, un $70 \%$ hipertensión arterial, un $40 \%$ tenían ojos claros y un $35 \%$ habían sido sometidos a cirugía previa de cataratas, destacando que un 95\% de los mismos presentaban niveles de colesterol elevados en sangre. 
Evaluando conjuntamente los factores asociados en ambas patologías EA-DMAE, observamos que en un $66,7 \%$ de la muestra coincidía la exposición solar y en un $41,7 \%$ la cirugía previa de cataratas. La hipertensión arterial aparece en el 75\% de los casos y la hipercolesterolemia en el 91,7\%.

Por último, y de acuerdo a la hipótesis de trabajo planteada, se obtuvo un resultado positivo que determina relación causa-efecto (Odds ratio). En la tabla de contingencia (tabla II) se observa cómo el porcentaje del grupo de «casos» que presentan DMAE $(42,4 \%)$ es superior al del grupo de «controles» que también la presentan $(25 \%)$.

Por otro lado la Chi cuadrado de Pearson, aunque no establece diferencias significativas, al aplicar el estadístico exacto de Fisher obtenemos una significación exacta bilateral EA-DMAE de 0,649, indicativa de protección en el grupo control, diferencia que, o bien puede ser cierta de acuerdo a la hipótesis planteada, o bien puede ser errónea de acuerdo a la diferencia entre las edades de los grupos control y experimental.

\section{DISCUSIÓN}

Con relación a los factores de riesgo para el desarrollo, tanto de EA como de DMAE, algunos autores (13-15) han considerado al tabaco, aunque en nuestro estudio no aparece dicha relación, que tampoco aparece en relación a la presencia de ojos claros. Sí existe coincidencia con otros autores (16-19) entre el colesterol elevado y ambas patologías, haciendo responsable a la Apo E como proteína transportadora de colesterol (20); además, también coincidimos con ellos en la presencia de otros factores de riesgo como la hipertensión arterial, la cardiopatía isquémica y la exposición solar para ambas patologías. La relación que tiene la DMAE con la exposición solar y la cirugía previa de cataratas es directamente proporcional $(21,22)$.

Diferentes estudios $(13,15,16)$ constataron que la arterioesclerosis y el tabaco unidos potencian su

Tabla II. Relación entre los grupos casos y controles y la presencia o no de DMAE

\begin{tabular}{lrll}
\hline & \multicolumn{2}{c}{ DMAE } & Total \\
& Sí & No & \\
\hline Casos (demencia) & $14(42,4 \%)$ & $19(57,6 \%)$ & $33(100 \%)$ \\
Controles & $6(25 \%)$ & $18(75 \%)$ & $24(100 \%)$ \\
Total & $20(35,1 \%)$ & $37(64,9 \%)$ & $57(100 \%)$ \\
\hline \hline
\end{tabular}

efecto neurodegenerativo. La arterioesclerosis $(20,23,24)$ produce un engrosamiento de la membrana de Bruch en la DMAE y un aumento en la angiopatía amiloide en la EA, condicionantes de la disminución del flujo vascular y de daño endotelial (25), mientras que el tabaco genera neurotoxicidad al disminuir el aporte de oxígeno a los tejidos.

El estudio de Rótterdam (16) describe al alelo E4 de la Apo Lipoproteína E como asociado a ambos desórdenes neurodegenerativos, pero de manera opuesta, significando esto que parece proteger de la DMAE $(26,27)$ pero no así de la EA $(28,29)$. La presencia de esta contraposición hace improbable que la apolipoproteína del genotipo E pueda contribuir a establecer una asociación entre la DMAE y EA, teniendo que determinar otros factores patogénicos neurodegenerativos comunes.

El estudio de Rotterdam (16) calculó la incidencia de la EA para las fases sucesivas de la degeneración macular, observándose una relación directa, con un alto riesgo de incidencia para el desarrollo posterior de EA en las fases 3 y 4 de la DMAE.

Blanks $(17,30)$ comparó retinas de una población mayor con y sin EA, considerando el número de células de la capa ganglionar, su distribución, densidad, proyecciones centrales y propiedades fisiológicas, observando los defectos tempranos de la retina en la población con Alzheimer.

Johnson (18), justifica la relación entre la EA y la DMAE, estableciendo que los depósitos de drusas contienen una variedad de moléculas inmunomoduladoras que desencadenan eventos inflamatorios locales y activan la cascada de complemento, degenerando las células de los fotorreceptores. Postulan que este proceso inflamatorio crónico es el elemento patogénico primario más importante de la DMAE. El $\beta$-amiloide se ha implicado como el activador principal, ya que forma parte estructural del componente vesicular dentro de la drusa. Este proceso de activación del complemento desencadena la atrofia del epitelio pigmentario de la retina, la generación de drusas y la patogenia de la DMAE.

Anderson $(6,19)$ demuestra que la herencia de la apolipoproteína E4 va unida tanto a la EA, como a la DMAE, estableciendo una relación protectora de la apolipoproteína E4 para la DMAE exudativa, pero de riesgo para el EA, por lo que es necesario buscar otros factores patogénicos comunes; así como, también, que la acumulación de drusas en la mácula es el factor de riesgo más importante para el desarrollo de DMAE. Los resultados de sus estu- 
dios establecieron que los remanentes celulares derivados de las células del epitelio pigmentario de la retina, depositados entre la lamina basal de la misma y la membrana de Bruch contribuían al estimulo inflamatorio crónico y a una posterior formación de drusas, coincidiendo con otros autores (31). Este hecho, podría conducir a la pérdida de la visión progresiva en la porción central de la mácula. Resultados que apoyan a la inflamación local como un papel importante en la génesis de las drusas y sugieren que este proceso es similar al que ocurre en otras enfermedades relacionadas con la edad, como la EA y la arteriosclerosis.

La relación funcional entre las drusas y la DMAE ha sido y continúa siendo motivo de controversia, por considerarse, por un lado, que la formación de las drusas es un proceso normal en el envejecimiento, mientras que por el otro se establece que constituyen una etapa temprana de la degeneración macular atrófica o exudativa. Muchos de los acúmulos proteicos asociados a las drusas han sido identificados como responsables en la patogenia de otras enfermedades como la EA, arteriosclerosis y amiloidosis (32), abriendo la posibilidad de orígenes comunes. La Apo E es una proteína transportadora de colesterol que está presente en las drusas duras y blandas (6,33); en el cerebro una de las funciones de la Apo E es la de regular el colesterol durante el proceso de remodelación neuronal. La herencia del alelo E de la apolipoproteína está implicada en la patogenia de la arteriosclerosis y de la EA (34); pero con un riesgo disminuido de DMAE exudativa.

Existen por tanto evidencias suficientes para pensar que la EA y la DMAE tienen factores etiológicos y patogénicos comunes, y aunque en nuestro estudio no hemos podido establecer una relación significativa entre ambas patologías, posiblemente en base al tamaño de la muestra o a las diferencias de edades entre ambos grupos, creemos establecida la asociación para que en futuros trabajos quede demostrada significativamente, abriendo un nuevo campo de investigación que de alguna manera permita utilizar criterios de degeneración retiniana como pronósticos de las demencias tipo Alzheimer.

\section{BIBLIOGRAFÍA}

1. Klein R, Klein BE, Linton KL. Prevalence of age-related maculopathy. The Beaver Dam Eye Study. Ophthalmology 1992; 99: 933-943.
2. Klein R, Klein BE, Jensen SC, Meuer SM. The five-year incidence and progression of age-related maculopathy. The Beaver Dam Eye Study. Ophthalmology 1997; 104: 7-21.

3. Dentchev T, Milam AH, Lee VM, Trojanowski JQ, Dunaief $J L$. Amyloid-beta is found in drusen from some age-related macular degeneration retinas, but not in drusen from normal retinas. Mol Vis 2003; 9: 184-190.

4. Vingerling JR, Dielemans I, Hofman A, Grobbee DE, Hijmering $M$, Kramer $C F$, et al. The Prevalence of age-related maculopathy in the Rotterdam Study. Ophthalmology 1995; 102: 205-210.

5. Morris JC, Storandt M, McKeel DW Jr, Rubin EH, Price $J L$, Grant EA, et al. Cerebral amyloid deposition and diffuse plaques in "normal" aging: Evidence for presymptomatic and very mild Alzheimer's disease. Neurology 1996; 46: 707-719.

6. Anderson DH, Ozaki S, Nealon M, Neitz J, Mullins RF, Hageman GS, et al. Local cellular sources of apolipoprotein $E$ in the human retina and retinal pigmented epithelium: implications for the process of drusen formation. Am J Ophthalmol 2001; 131: 767-781.

7. Corder EH, Saunders AM, Strittmatter WJ, Schmechel DE, Gaskell PC, Small GW, et al. Gene dose of apolipoprotein e type 4 allele and the risk of Alzheimer's disease in late onset families. Science 1993; 261: 921-923.

8. Tang MX, Stern Y, Marder K, Bell K, Gurland B, Lantigua $R$, et al. The APOE epsilon 4 allele and the risk of Alzheimer disease among African Americans, whites, and Hispanics. JAMA 1998; 279: 751-755.

9. Saunders AM, Strittmatter WJ, Schmechel D, St GeorgeHyslop PH, Pericak-Vance MA, Joo SH, et al. Association of apolipoprotein $E$ allele epsilon 4 with late-onset familial and sporadic Alzheimer's disease. Neurology 1993; 43: 1467-1472.

10. McKhann G, Drachman D, Folstein M, Katzman R, Price $D$, Stadlan EM. Clinical diagnosis of Alzheimer's disease: report of the NINCDS-ADRDA Work Group under the auspices of Department of Health and Human Services Task Force on Alzheimer's Disease. Neurology 1984; 34 : 939-944.

11. Asociación Americana de Psiquiatria. Manual diagnóstico y estadístico de los trastornos mentales. Versión española de la 4th edición, Texto Revisado (DSM-IV-TR). Barcelona: Masson SA; 2002; 179-180.

12. Bird AC, Bressler NM, Bressler SB, Chisholm IH, Coscas $G$, Davis MD, et al. An international classification and grading system for age-related maculopathy and age-related macular degeneration. The International ARM Epidemiological Study Group. Surv Ophthalmol 1995; 39: $367-$ 374.

13. Seddon JM, Willett WC, Speizer FE, and Hankinson SE. A prospective study of cigarette smoking and age-related macular degeneration in women. JAMA 1996; 276: 1141 1146.

14. Klein R, Klein BE, Moss SE. Relation of smoking to the incidence of age-related maculopathy. The Beaver Dam Eye Study. Am J Epidemiol 1998; 147: 103-110.

15. Ott A, Slooter AJ, Hofman A, van Harskamp F, Witteman $J C$, Van Broeckhoven C, et al. Smoking and risk of dementia and Alzheimer's disease in a population-based cohort study: the Rotterdam Study. Lancet 1998; 351: 18401843. 
16. Klaver CC, Ott A, Hofman A, Assink JJ, Breteler MM, de Jong PT. Is age-related maculopathy associated with Alzheimer's disease? The Rotterdam Study. Am J Epidemiol 1999; 150: 963-968.

17. Blanks JC, Torigoe Y, Hinton DR, Blanks RH. Retinal degeneration in the macula of patients with Alzheimer's disease. Ann N Y Acad Sci 1991; 640: 44-46.

18. Johnson LV, Leitner WP, Rivest AJ, Staples MK, Radeke MJ, Anderson DH. The Alzheimer's A beta-peptide is deposited at sites of complement activation in pathologic deposits associated with aging and age-related macular degeneration. Proc Natl Acad Sci USA 2002; 99: 11830-11835.

19. Anderson DH, Mullins RF, Hageman GS, Johnson LV. A role for local inflammation in the formation of drusen in the aging eye. Am J Ophthalmol 2002; 134: 411-431.

20. Hofman A, Ott A, Breteler MM, Bots ML, Slooter AJ, van Harskamp $F$, et al. Atherosclerosis, apolipoprotein E, and prevalence of dementia and Alzheimer's disease in the Rotterdam Study. Lancet 1997; 349: 151-154.

21. Hyman L, Schatat AP, He Q, Leske MC. Hypertension, cardiovascular disease, and age-related macular degeneration. Arch Ophthalmol 2000; 118: 351-358.

22. Hirvela H, Luukinen H, Laara E, Sc L, Laatikainen L. Risk factors of age-related maculopathy in a population 70 years of age or older. Ophthalmology 1996; 103: 871-878.

23. Vingerling JR, Dielemans I, Bots ML, Hofman A, Grobbee DE, Jong PTV. Age-related macular degeneration is associated with atherosclerosis. The Rotterdam Study. Am J Epidemiol 1995; 142: 404-409.

24. Pauleikhoff D, Chen JC, Chisholm IH, Bird AC. Choroidal perfusion abnormality with age-related Bruch's membrane change. Am J Ophthalmol 1990; 109: 211-217.

25. Ellis RJ, Olichney JM, Thal LJ, Mirra SS, Morris JC, Beekly D, et al. Cerebral amyloid angiopathy in the brains of patients with Alzheimer's disease: the CERAD experience, Part XV. Neurology 1996; 46: 1592-1596.
26. Souied EH, Benlian P, Amouyel P, Feingold J, Lagarde $J P$, Munnich A, et al. The epsilon 4 allele of the apolipoprotein e gene as a potential protective factor for exudative age-related macular degeneration. Am J Ophthalmol 1998; 125: 353-359.

27. Ott A, Breteler MM, van Harskamp F, Stijnen T, Hofman A. Incidence and risk of dementia. The Rotterdam Study. Am J Epidemiol 1998; 147: 574-580.

28. Strittmatter WJ, Saunders AM, Schmechel D, PericakVance M, Enghild J, Salvesen GS, et al. Apolipoprotein E: high-avidity binding to beta-amyloid and increased frequency of type 4 allele in late-onset familial Alzheimer disease. Proc Natl Acad Sci USA 1993; 90: 19771981.

29. Tsai MS, Tangalos EG, Petersen RC, Smith GE, Schaid $D J$, Kokmen E, et al. Apolipoprotein E: risk factor for Alzheimer disease. Am J Hum Genet 1994; 54: 643-649.

30. Blanks JC, Hinton DR, Sadun AA, Miller CA. Retinal ganglion cell degeneration in Alzheimer's disease. Brain Res 1989; 501: 364-372.

31. Penfold PL, Madigan MC, Gillies MC, Provis JM. Immunological and aetiological aspects of macular degeneration. Prog Retin Eye Res 2001; 20: 385-414.

32. Mullins RF, Russell SR, Anderson DH, Hageman GS. Drusen associated with aging and age-related macular degeneration contain proteins common to extracellular deposits associated with atherosclerosis, elastosis, amyloidosis, and dense deposit disease. FASEB J. 2000; 14: 835846.

33. Klaver CC, Kliffen M, van Duijn V, Hofman A, Cruts M, Grobbee DE, et al. Genetic association of apolipoprotein $E$ with age-related macular degeneration. Am J Hum Genet 1998; 63: 200-206.

34. Davignon J, Cohn JS, Mabile L, Bernier L. Apolipoprotein $E$ and atherosclerosis: insight from animal and human studies. Clin Chim Acta 1999; 286: 115-143. 